\title{
ON EXTREME POINTS OF THE JOINT NUMERICAL RANGE
}

\author{
YOUNGOH YANG
}

\begin{abstract}
Our purpose is to study the relationship between the joint numerical range and joint essential numerical range. We give an example of an operator such that the set of all extreme points of the closure of its essential numerical range is not a subset of the set of all extreme points of its numerical range. We shall investigate the extreme points of a convex joint essential numerical range.
\end{abstract}

J.S. Lancaster [11] showed that the set of all extreme points of the closure of the numerical range is a subset of the union of the essential numerical range and numerical range. S.C. Arora and R. Kumar [1] proved this result for a convex joint numerical range. By means of this result, our purpose is to study the relationship between the joint numerical range and joint essential numerical range.

G. Garske [7] has investigated the extreme point of the closure of the numerical range. R. Kumar [10] has studied it in case of 2-tuple of operators. We give an example of an operator such that the set of all extreme points of the closure of its essential numerical range generally is not a subset of the set of all extreme points of its numerical range. In this paper, we shall investigate the extreme points of the essential numerical numerical range of an operator and prove this result for a convex joint essential numerical range.

Let $H$ be an infinite dimensional complex Hilbert space with the scalar product $($, ) and the norm $\|\cdot\|$. All operators will be assumed to linear and bounded. $K(H)$ denotes the ideal of compact operators on $H$. Let $A=\left(A_{1}, A_{2}\right)$ be a 2-tuple of operators on $H$. The joint numerical range $W(A)$ of $A$ is defined as

$$
W(A)=\left\{\left(\left(A_{1} x, x\right),\left(A_{2} x, x\right)\right): x \in H,\|x\|=1\right\} .
$$

$W(A)$ is a bounded subset of $\mathbf{C}^{2}$ and is in general not convex. However for 2-tuple $A=\left(A_{1}, A_{2}\right)$ of commuting normal operators, $W(A)$ is convex [6]. as

If $T$ is any operator on $H$, then the essential numerical range $W_{e}(T)$ of $T$ is defined

$$
W_{e}(T)=\cap\{\overline{W(T+K)}: K \in K(H)\} .
$$

Received October 17, 1989.

1980 Mathematics Subject Classification. Primary 47A12, Secondary 47A10.

Key words and phrases. Joint numerical range, joint essential numerical range, joint eigenvalue. 
Definition ([1]). The joint essential numerical range $W_{e}(A)$ of $A=\left(A_{1}, A_{2}\right)$ is defined as

$$
W_{e}(A)=\cap\left\{\overline{W\left(A_{1}+K_{1}, A_{2}+K_{2}\right)}: K_{1}, K_{2} \in K(H)\right\},
$$

where $W\left(A_{1}+K_{1}, A_{2}+K_{2}\right)$ denotes the joint numerical range of $\left(A_{1}+K_{1}, A_{2}+K_{2}\right)$. $W_{e}(A)$.

We note that $W_{e}(A) \subset \overline{W(A)}$ and $W_{e}\left(A^{*}\right)=\left[W_{e}(A)\right]^{*}$, the complex conjugate of

Let $\operatorname{Ext} X$ denote the set of all extreme points of a connected set $X$ and co $Y$ denote the convex hull of the set $Y$. If $T$ is any operator on $H$, J.S. Lancaster [11] proved that

$$
\mathbb{E x t} \overline{W(T)} \subset W_{e}(T) \cup W(T)
$$

S.C. Arora and R. Kumar [1] generalized this result in the following:

Theorem 1 ([1]). Assume that $W(A)$ is convex. Then $\operatorname{Ext} \overline{W(A)} W_{e}(A) \cup W(A)$.

Proof. See Theorem 1.4 in [1].

Corollary 1. Assume that $W(A)$ is convex. Then

$$
\overline{W(A)}=\overline{\operatorname{co}}\left(W_{e}(A) \cup W(A)\right)
$$

where $\overline{\mathrm{CO}} X$ denotes the closed convex hull of the set $X$.

Proof. Since $W(A)$ is convex, $\overline{W(A)}$ is also convex. So by the Krein-Milman Theorem and Theorem 1,

$$
\overline{W(A)}=\overline{\operatorname{co}}[\operatorname{Ext} \overline{W(A)}] \subset \overline{\operatorname{co}}\left[W_{e}(A) \cup W(A)\right] .
$$

Also $W_{e}(A) \subset \overline{W(A)}$, and so $W_{e}(A) \cup W(A) \subset \overline{W(A)}$. Hence since $\overline{W(A)}$ is convex, $\operatorname{co}\left(W_{e}(A) \cup W(A)\right) \subset \overline{W(A)}$. Therefore $\overline{W(A)}=\overline{c o}\left(W_{e}(A) \cup W(A)\right)$.

Corollary 2. Assume that $W(A)$ is convex. Then $W(A)$ is closed if and only if $W_{e}(A) \subset W(A)$.

Proof. Assume that $W(A)$ is closed. Then $W_{e}(A) \subset W(A)$. Conversely if $W_{e}(A) \subset$ $W(A)$, then by Theorem 1 ,

$$
\operatorname{Ext} \overline{W(A)} \subset W_{e}(A) \cup W(A)=W(A)
$$

Hence $\operatorname{Ext} \overline{W(A)} \subset \operatorname{Ext} W(A)$. Since $W(A)$ is convex, $\overline{W(A)}=W(A)$.

Corollary 3. Assume that $W(A)$ is convex. If Ext $W(A)$ is a subset of $W_{e}(A)$, then $\overline{W(A)}=$ co $W_{e}(A)$.

Proof. Let $\lambda \in \operatorname{Ext} \overline{W(A)}$. If $\lambda \in W(A)$, If $\lambda \in W(A)$, then $\lambda \in \operatorname{Ext} W(A) \subset$ $W_{e}(A)$. If $\lambda \notin W(A)$, then by Theorem $1, \lambda \in W_{e}(A)$. Hence Ext $\overline{W(A)} \subset W_{e}(A)$. 
Taking convex hulls, we get $\overline{W(A)}=\overline{\operatorname{co}}[\operatorname{Ext} \overline{W(A)}] \subset \overline{\operatorname{co}} W_{e}(A)=\operatorname{co} W_{e}(A) \subset \operatorname{co} \overline{W(A)}=$ $\overline{W(A)}$. Therefore $\overline{W(A)}=\operatorname{co} W_{e}(A)$.

For example, if $A=\left(A_{1}, A_{2}\right)$ is a 2-tuple of Toeplitz operators, then the joint numerical range $W(A)$ of $A$ is convex (See Theorem 2.6 of [6]) and is open unless it consists of only one point (See Proposition 3 of [3]). So by Corollary $3, \overline{W(A)}=\operatorname{co} W_{e}(A)$ for a 2-tuple $A=\left(A_{1}, A_{2}\right)$ of Toeplitz operators.

A 2 -tuple $\lambda=\left(\lambda_{1}, \lambda_{2}\right)$ of complex numbers is called a joint eigenvalue of finite multiplicity for $A$ if $\operatorname{dim} N(A-\lambda I)<\infty$ where $N(A-\lambda I)=\left\{x \in H:\left(A_{1}-\lambda_{1} I\right) x=\right.$ $\left.\left(A_{2}-\lambda_{2} I\right) x=0\right\}$.

P. Juneja [8] has proved that if $A=\left(A_{1}, A_{2}\right)$ is a 2-tuple of commuting normal operators and if $\lambda=\left(\lambda_{1}, \lambda_{2}\right)$ is an extreme point of $\overline{W(A)}$ and $\lambda \in W(A)$, then $\lambda$ is a reducing joint eigenvalue. However S.C. Arora and R. Kumar [1] has proved that if $A=\left(A_{1}, A_{2}\right)$ is a 2-tuple of commuting normal operators and if $\lambda=\left(\lambda_{1}, \lambda_{2}\right)$ is an extreme point of $\overline{W(A)}$, then either $\lambda \in W_{e}(A)$ or $\lambda$ is a reducing joint eigenvalue of finite multiplicity for $A$. We generalize this result in the following :

Corollary 4. If $A=\left(A_{1}, A_{2}\right)$ is a 2-tuple of doubly commuting hyponormal operators on $H, W(A)$ is convex and $\lambda=\left(\lambda_{1}, \lambda_{2}\right)$ is an extreme point of $\overline{W(A)}$, then either $\lambda \in W_{e}(A)$ or $\lambda$ is a joint eigenvalue of finite multiplicity.

Proof. By Theorem 1, Ext $\overline{W(A)} \subset W_{e}(A) \cup W(A)$. If $\lambda \in W_{e}(A)$, there is nothing to prove. If $\lambda \notin W_{e}(A)$, then $\lambda \in W(A)$ and so $\lambda$ must be an extreme point of $W(A)$. By Theorem 1 [4], $\lambda$ is a joint eigenvalue of $A$. Since $\sigma_{e}(A) \subset W_{e}(A)$ (See Corollary 1.3 of [1]), $\lambda \notin \sigma_{e}(A)$ and therefore there exist operators $B_{1}$ and $B_{2}$ such that $B_{1}\left(A_{1}-\lambda_{1} I\right)+B_{2}\left(A_{2}-\lambda_{2} I\right)=F$ for some Fredholm operator $F$. Now for each $x \in N(A-\lambda I)=\left\{x \in H:\left(A_{1}-\lambda_{1} I\right) x=\left(A_{2}-\lambda_{2} I\right) x=0\right\}, F(x)=0$. Since $F$ has finite dimensional null space, $\lambda$ is a joint eigenvalue of finite multiplicity.

Example. Let $T: L^{2}([-1,1]) \rightarrow L^{2}([-1,1])$ be the selfadjoint multiplication operator defined by $(T f)(t)=t f(t)$ for $f \in L^{2}([-1,1]), t \in[-1,1]$, and $S=T \oplus 2 I_{C} n$ where $I_{\mathbf{C}} n$ denotes the identity operator on the $n$-dimensional unitary space $\mathbb{C}^{n}$. Then $I_{C} n$ is compact and $W(S)=\operatorname{co}\left(W(T) \cup W\left(2 I_{\mathbf{C}} n\right)\right)=\operatorname{co}((-1,1) \cup\{2\})=(-1,2]$. Also $W_{e}(S)=\operatorname{co}\left(W_{e}(T) \cup W_{e}\left(2 I_{C} n\right)\right)=\operatorname{co}\left(W_{e}(T) \cup\{0\}\right) \subset[-1,1]$. Hence $\operatorname{Ext} \overline{W(S)}=$ $\{-1,2\}$, and so $\operatorname{Ext} W_{e}(S) \not \subset \operatorname{Ext} \overline{W(S)}$.

In [7], G. Garske proved that if $\lambda$ is an extreme point of $\overline{W(T)}$, the following statement $\left(^{*}\right)$ is true:

(*) Let $\left\{x_{n}\right\}$ be a sequence of unit vectors in $H$ weakly converging to $x \in H$ such that $\left(T x_{n}, x_{n}\right) \rightarrow \lambda$. then either $x=0$ or $\left(T \frac{x}{\|x\|}, \frac{x}{\|x\|}\right)=\lambda$.

Motivated by the above example and G. Garske's result [7], we give the following.

Theorem 2. Let $\lambda$ be an extreme point of the essential numerical range $W_{e}(T)$ of an operator $T$ on $H$. Then the following is true:

Let $\left\{x_{n}\right\}$ be a sequence of unit vectors in $H$ weakly convergent to $x \in H$ such that 
$\left((T+K) x_{n}, x_{n}\right) \rightarrow \lambda$ for any $K$ in $K(H)$. Then either $x=0$ or $\left((T+K) \frac{x}{\|x\|}, \frac{x}{\|x\|}\right)=\lambda$ for any $K$ in $K(H)$.

Proof. Let $y_{n}=x_{n}-x$. Then $y_{n} \rightarrow 0$ weakly and $\left\|y_{n}\right\| \leq 2$ since $\|x\| \leq 1$. So we may assume by passing to a subsequence, if necessary, that there is a real number $\epsilon \geq 0$ such that $\left\|y_{n}\right\| \varepsilon$. Now $1=\left\|x_{n}\right\|^{2}=\left\|y_{n}\right\|^{2}+2 \operatorname{Re}\left(y_{n}, x\right)+\|x\|^{2}$. Let $K$ be any compact operator. Then

$$
\begin{aligned}
\left((T+K) x_{n}, x_{n}\right)= & \left(T y_{n}, y_{n}\right)+\left(y_{n}, T^{*} x\right)+\left(T x, y_{n}\right)+(T x, x) \\
& +\left(K y_{n}, y_{n}\right)+\left(y_{n}, K^{*} x\right)+\left(K x, y_{n}\right)+\left(K x, y_{n}\right)+(K x, x) .
\end{aligned}
$$

Since $y_{n} \rightarrow 0$ weakly, $K y_{n} \rightarrow 0$ strongly, and $\left.((T+K)) x_{n}, x_{n}\right) \rightarrow \lambda$, we see that $1=\epsilon^{2}+\|x\|^{2}$ and $\left.\left(T y_{n}, y_{n}\right) \rightarrow \lambda-((T+K)) x, x\right)$. If $\epsilon=0$, then $\|x\|=1$ and $\lambda=((T+K) x, x)$ for any $K \in K(H)$ since $\left(T y_{n}, y_{n}\right) \rightarrow 0$. Suppose $\epsilon \neq 0$. For every $K$ in $K(H)$, we let

$$
\begin{aligned}
\alpha & =\left((T+K) \frac{x}{\|x\|}, \frac{x}{\|x\|}\right) \text { for } x \neq 0 \text { and } \\
\beta_{n} & =\left((T+K) \frac{y_{n}}{\left\|y_{n}\right\|}, \frac{y_{n}}{\left\|y_{n}\right\|}\right) \text { for all } n \text { such that } y_{n} \neq 0 .
\end{aligned}
$$

Then for each $K \in K(H), \alpha \in W(T+K)$ and $\left\{\beta_{n}\right\}$ is a sequence in $W(T+K)$ which converges to some $\beta \in \overline{W(T+K)}$ such that $\beta=\frac{\lambda}{\epsilon^{2}}-\frac{\|x\|^{2}}{\epsilon^{2}} \alpha$.

This means that $\lambda=\epsilon^{2} \beta+\|x\|^{2} \alpha$. Since $\epsilon^{2}+\|x\|^{2}=1, \lambda$ lies on the line segement from $\alpha$ to $\beta$. Since $\lambda$ is an extreme point of $W_{e}(T)$, we conclude $\lambda=\alpha$ or $\lambda=\beta$. In the latter case, we have $\lambda=\epsilon^{2} \lambda+\|x\|^{2} \alpha$ i.e., $\left(1-\epsilon^{2}\right) \lambda=\|x\|^{2} \alpha$, and this gives $\lambda=\alpha=\left((T+K) \frac{x}{\|x\|}, \frac{x}{\|x\|}\right)$ for any $K$ in $K(H)$.

In [10], R. Kumar proved the above result $\left({ }^{*}\right)$ for a convex joint numerical range $W(A)$ of $A=\left(A_{1}, A_{2}\right)$, by means of Garske's method. In [2], M. Chō and M. Takaguchi proved the result $\left(^{*}\right)$ in the case that $\lambda$ is a bare point of $\overline{W(A)}$ for any 2-tuple $A=$ $\left(A_{1}, A_{2}\right)$ of operators.

The following theorem is an extension of the above theorem to joint essential numerical range.

Theorem 3. Suppose that the joint essential numerical range $W_{e}(A)$ of $A=$ $\left(A_{1}, A_{2}\right)$ is convex and $\lambda=\left(\lambda_{1}, \lambda_{2}\right)$ is an extreme point of $W_{e}(A)$. Then the following statement is true :

Let $\left\{x_{n}\right\}$ be a sequence of unit vectors in $H$ weakly converging to $x \in H$ such that

$$
\left(\left(A_{i}+K_{i}\right) x_{n}, x_{n}\right) \rightarrow \lambda_{i} \text { for any } K \in K(H)(i=1,2) .
$$

Then either $x=0$ or $\left(\left(T_{i}+K_{i}\right) \frac{x}{\|x\|}, \frac{x}{\|x\|}\right)=\lambda_{i}$ for any $K$ in $K(H)(i=1,2)$.

Proof. The proof is similar to that of Theorem 2. 


\section{References}

[1] S.C. Arora \& R. Kumar, "Joint essential numerical range," Glasnik Mat. Ser III 18 (38) (1983),
$31.7-320$.

[2] M. Chō \& M. Takaguchi, "Boundary of joint numerical range," Sci. Rep. Hirosaki Univ., 26 (1979),
$69-71$.

[3] — - "Boundary points of joint numerical ranges," Pacific J. Math., 95(1) (1981), 27-35.

[4] - "On extreme points of the joint numerical range of several hyponormal operators," Glasnik
Mat. Ser III 19 (39) (1984), 285-292.

[5] J. B. Conway, A course in functional analysis, Springer-Verlag NY Inc. 1985.

[6] A.T. Dash, "Joint numerical range," Glasnik Mat. Ser III 7 (27) (1972), 75-81. [7] G. Garske, "The boundary of the numerical range of an operator," J. Math. Anal. Appl. 68 (1979),
$605-607$. [8] P. Juneja, "On extreme points of the joint numerical range of commuting normal operators," Pacific
J. Math. 67 (2) (1976), 473-476.

[9] E. M. Klein, "The numerical range of a Toeplitz operator," Proc. Amer. Math. Soc., 35(1) (1972),
101-103. [10] R. Kumar, "A note on the boundary of the joint numerical range," Tamkang J. Math., 14 (1)
(1983), 1-4. [11] J.S. Lancaster, "The boundary of the numerical range," Proc. Amer. Math. Soc., 49(2) (1975),
393-398.

Dept. of Mathematics, Cheju National University, Cheju-do, 690-756, Korea. 